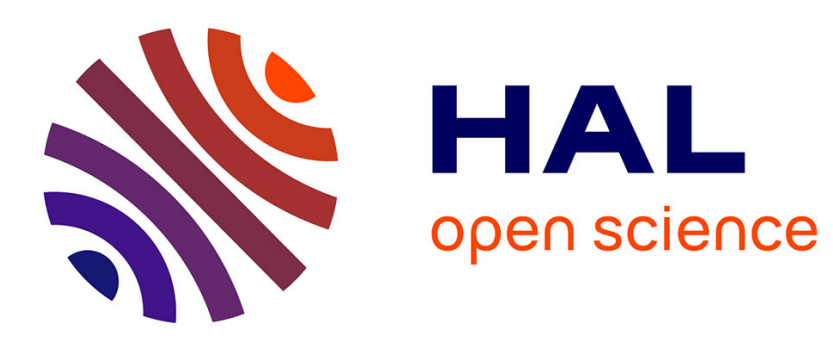

\title{
Distribution of Hecke eigenvalues of newforms in short intervals
}

\author{
Jie Wu, Wenguang Zhai
}

\section{To cite this version:}

Jie Wu, Wenguang Zhai. Distribution of Hecke eigenvalues of newforms in short intervals. Quarterly Journal of Mathematics, 2013, 64 (2), pp.619-644. 10.1093/qmath/has007 . hal-01278937

\section{HAL Id: hal-01278937 \\ https://hal.science/hal-01278937}

Submitted on 25 Feb 2016

HAL is a multi-disciplinary open access archive for the deposit and dissemination of scientific research documents, whether they are published or not. The documents may come from teaching and research institutions in France or abroad, or from public or private research centers.
L'archive ouverte pluridisciplinaire HAL, est destinée au dépôt et à la diffusion de documents scientifiques de niveau recherche, publiés ou non, émanant des établissements d'enseignement et de recherche français ou étrangers, des laboratoires publics ou privés. 


\title{
DISTRIBUTION OF HECKE EIGENVALUES OF NEWFORMS IN SHORT INTERVALS
}

\author{
JIE WU AND WENGUANG ZHAI
}

\begin{abstract}
We explore nonvanishing of Hecke eigenvalues in short arithmetic progressions and their signs in short intervals by further developing $\mathscr{B}$-free number method and studying moments in short intervals. We improve some results of Alkan \& Zaharescu [3] and Lau \& Wu [20] on the distribution of Hecke eigenvalues.
\end{abstract}

\section{INTRODUCTION}

The distribution of Fourier coefficients of holomorphic cusp forms is one of central questions in modular form theory. The well known Lang-Trotter conjecture suggests that these coefficients vanish rarely over prime variables in a precise sense. When the forms are primitive, according to Sato-Tate's conjecture their coefficients restricted in prime variables are equi-distributed on $[-2,2]$ with respect to the SatoTate measure. In [18], Kowalski, Robert \& Wu used Rankin-Selberg's theory and $\mathscr{B}$-free number method to investigate the nonvanishing or lacunary of these coefficients. Recently Lau \& Wu [20] further studied sign changes of Hecke eigenvalues. The present work is a continuation of $[18,20]$. Here we shall explore nonvanishing of these coefficients in short arithmetic progressions and their signs in short intervals by further developing $\mathscr{B}$-free number method and studying moments in short intervals.

Let us begin by fixing our notation. For any integers $k \geqslant 1, N \geqslant 1$ and any Dirichlet character $\chi$ modulo $N$ verifying $\chi(-1)=(-1)^{k}$, we denote by $\mathcal{S}_{k}(N, \chi)$ the vector space of holomorphic cusp forms of weight $k$ for the Hecke congruence group $\Gamma_{0}(N)$ with nebentypus $\chi$. When $\chi$ is the trivial character modulo $N$, we simply write $\mathcal{S}_{k}(N)$. We also denote by $\mathcal{S}_{k}^{*}(N, \chi)$ (resp. $\left.\mathcal{S}_{k}^{*}(N)\right)$, the set of all normalized Hecke eigencuspforms in $\mathcal{S}_{k}(N, \chi)$ (resp. $\mathcal{S}_{k}(N)$ ). Here the normalization is taken in the way that the Fourier series expansion of $f \in \mathcal{S}_{k}^{*}(N, \chi)$ at the cusp $\infty$,

$$
f(z)=\sum_{n=1}^{\infty} \lambda_{f}(n) n^{(k-1) / 2} \mathrm{e}^{2 \pi \mathrm{i} n z} \quad(\Im m z>0),
$$

has its first coefficient equal to one (i.e. $\lambda_{f}(1)=1$ ). Inherited from the Hecke operators, the normalized Fourier coefficient $\lambda_{f}(n)$ satisfies the following relation

$$
\lambda_{f}(m) \lambda_{f}(n)=\sum_{d \mid(m, n)} \chi(d) \lambda_{f}\left(\frac{m n}{d^{2}}\right)
$$

Date: June 30, 2013.

2000 Mathematics Subject Classification. 11F12; 11F30; 11F66; 11L15; 11N25.

Key words and phrases. Fourier coefficients of modular forms, Cusp form, Eigenvalue, Voronoi's formula, sign change, exponential sums, $\mathscr{B}$-free numbers. 
for all integers $m \geqslant 1$ and $n \geqslant 1$. In particular, $\lambda_{f}(n)$ is multiplicative. According to Deligne [7], if $f \in \mathcal{S}_{k}^{*}(N)$ we have

$$
\left|\lambda_{f}(n)\right| \leqslant \tau(n)
$$

for all $n \geqslant 1$, where $\tau(n)$ is the divisor function.

1.1. Nonvanishing of Fourier coefficients of newforms in short progressions. Let $f \in \mathcal{S}_{k}^{*}(N, \chi)$ be a primitive cusp form not of CM type. One of Serre's main results in [23] states as follows:

$$
\left|\left\{p \leqslant x: \lambda_{f}(p)=0\right\}\right| \ll_{f, \delta} \frac{x}{(\log x)^{1+\delta}}
$$

for $x \geqslant 2$ and any $\delta<\frac{1}{2}$, from which he deduces that the series (1.1) is not lacunary, i.e. the set of indices $n$ where $\lambda_{f}(n) \neq 0$, has a positive density. Serre asked ([23], p. 183) for more precise statements. A stronger form of the problem is to find $y$ as small as possible (as a function of $x$, say $y=x^{\theta}$ with $\theta<1$ ) such that

$$
\left|\left\{x<n \leqslant x+y: \lambda_{f}(n) \neq 0\right\}\right| \gg_{f} y
$$

for $x \geqslant x_{0}(f)$.

Balog and Ono [4] apparently first noticed that this problem can be resolved by $\mathscr{B}$-free numbers theory, introduced by Erdős [9]. For a set of integers

$$
\mathscr{B}=\left\{b_{i}: 1<b_{1}<b_{2}<\ldots\right\}
$$

such that

$$
\sum_{i \geqslant 1} 1 / b_{i}<\infty \quad \text { and } \quad\left(b_{i}, b_{j}\right)=1 \quad(i \neq j),
$$

one says that $n \geqslant 1$ is $\mathscr{B}$-free if it is not divisible by any element in $\mathscr{B}$. Erdös [9] already showed in 1966 that there exists an absolute constant $\theta<1$ such that the interval $\left(x, x+x^{\theta}\right]$ contains a $\mathscr{B}$-free number for $x$ large enough. Now taking

$$
\mathscr{B}_{\mathfrak{P}_{f}}:=\mathfrak{P}_{f} \cup\left\{p^{2}: p \in \mathscr{P} \backslash \mathfrak{P}_{f}\right\}
$$

where $\mathscr{P}$ is the set of all primes and

$$
\mathfrak{P}_{f}:=\left\{p: p \mid N \text { or } \lambda_{f}(p)=0\right\}
$$

and using the fact that $\lambda_{f}(n)$ is multiplicative, we see that Erdös' result solves Serre's question in the affirmative. A quantitative result proving the analogue of (1.5) for general $\mathscr{B}$-free numbers was also obtained Szemerédi [24] as early as 1973. The best exponent is $7 / 17$, due to Kowalski, Robert \& Wu [18], where readers can find a detailed historical description on $\mathscr{B}$-free numbers in short intervals.

Alkan \& Zaharescu considered the analogue of (1.5) in short arithmetic progressions, by proving the following result ([3], Theorem 1): Suppose that $k \geqslant 2$ and $f \in \mathcal{S}_{k}^{*}(N, \chi)$ is a primitive form not of CM type. For every $\varepsilon>0, x \geqslant x_{0}(f, \varepsilon)$, $y \geqslant x^{9 / 20+(1803 / 10) \varepsilon}$ and $1 \leqslant a \leqslant q \leqslant x^{\varepsilon}$ with $(a, q)=1$, we have

$$
\mid\left\{x<n \leqslant x+y: n \equiv a(\bmod q) \text { and } \lambda_{f}(n) \neq 0\right\} \mid \gg_{f, \varepsilon} y / q .
$$

The analogue of (1.9) for general $\mathscr{B}$-free numbers has been established by the same authors [2, Theorem 1].

Here we propose a better and general result, by combining new estimates for exponential sums [30] and some ideas on $\mathscr{B}$-free numbers of [18]. 
Theorem 1. Suppose that $k \geqslant 2$ and $f \in \mathcal{S}_{k}^{*}(N, \chi)$ is a primitive form not of $C M$ type such that

$$
\left|\mathfrak{P}_{f} \cap[1, x]\right| \ll_{f} x^{\rho} \frac{(\log \log x)^{\Psi_{\rho}}}{(\log x)^{\Theta_{\rho}}} \quad(x \geqslant 2),
$$

where $\rho \in[0,1]$ and $\Theta_{\rho}, \Psi_{\rho}$ are real constants such that $\Theta_{1}>1$. Define

$$
\theta(\rho)= \begin{cases}29 \rho /(46 \rho+19) & \text { if } 190 / 323<\rho \leqslant 166 / 173 \\ 17 \rho /(26 \rho+12) & \text { if } 166 / 173<\rho \leqslant 1\end{cases}
$$

or

$$
\theta(\rho)=\frac{(\kappa+2) \rho}{(\kappa-\lambda+3) \rho+2} \quad \text { for } \quad \frac{\kappa}{\kappa-\lambda+1} \leqslant \rho \leqslant 1,
$$

where $(\kappa, \lambda)$ is an exponent pair. For every $\varepsilon>0, x \geqslant x_{0}(f, \varepsilon), y \geqslant x^{\theta(\rho)+100 \varepsilon}$ and $1 \leqslant a \leqslant q \leqslant x^{\varepsilon}$ with $(a, q)=1$, the inequality (1.9) holds.

According to (1.4), the hypothesis (1.10) holds with $\left(\rho, \Theta_{\rho}, \Psi_{\rho}\right)=\left(1, \frac{3}{2}-\delta, 0\right)$. Thus we immediately obtain the following corollary, which gives an improvement of Alkan \& Zaharescu's exponent 9/20 in (1.9).

Corollary 1. Suppose that $k \geqslant 2$ and $f \in \mathcal{S}_{k}^{*}(N, \chi)$ is a primitive form not of $C M$ type. For every $\varepsilon>0, x \geqslant x_{0}(f, \varepsilon), y \geqslant x^{17 / 38+100 \varepsilon}$ and $1 \leqslant a \leqslant q \leqslant x^{\varepsilon}$ with $(a, q)=1$, the inequality (1.9) holds.

The hypothesis (1.10) is known only when $\rho=1$, except for primitive forms $f \in \mathcal{S}_{2}^{*}(N)$ with integral coefficients. Those are associated to elliptic curves over $\mathbb{Q}$, and Elkies [8] has proved that (1.10) holds with $\left(\rho, \Theta_{\rho}, \Psi_{\rho}\right)=\left(\frac{3}{4}, 0,0\right)$. Theorem 1 implies the following corollary, which improves Alkan \& Zaharescu's exponent 9/22 ([3], Theorem 2).

Corollary 2. Let $f_{E}(z)=\sum_{n \geqslant 1} \lambda_{E}(n) \mathrm{e}(n z)$ be a primitive form associated with an elliptic curve $E / \mathbb{Q}$ without complex multiplication. For every $\varepsilon>0, x \geqslant x_{0}(E, \varepsilon)$, $y \geqslant x^{87 / 214+100 \varepsilon}$ and $1 \leqslant a \leqslant q \leqslant x^{\varepsilon}$ with $(a, q)=1$, the inequality (1.9) holds for $f=f_{E}$.

According to a result of David \& Pappalardi [6], the hypothesis (1.10) with $\lambda_{E}(n)$ defined as in Corollary 2 holds with $\left(\rho, \Theta_{\rho}, \Psi_{\rho}\right)=\left(\frac{1}{2}, 0,0\right)$ in average. By combining this with Theorem 1 with $(\kappa, \lambda)=\left(\frac{1}{6}, \frac{4}{6}\right)$, we obtain the following result of almost all type.

Corollary 3. Consider the family of elliptic curves $E_{c, d}: v^{2}=u^{3}+c u+d$ without complex multiplication, where $c$ and $d$ are integers with $|c| \leqslant C$ and $|d| \leqslant D$. Let

$$
f_{E_{c, d}}(z)=\sum_{n \geqslant 1} \lambda_{E_{c, d}}(n) \mathrm{e}(n z)
$$

be a primitive form associated with $E_{c, d}$ without complex multiplication. Let $X \rightarrow \infty$ be a parameter such that $\min \{C, D\} \geqslant X^{2+\eta}$ for some $\eta>0$. For every $\varepsilon>0$, $x \geqslant x_{0}\left(E_{c, d}, \varepsilon\right), y \geqslant x^{13 / 40+100 \varepsilon}$ and $1 \leqslant a \leqslant q \leqslant x^{\varepsilon}$ with $(a, q)=1$, the inequality (1.9) holds for $f=f_{E_{c, d}}$, with at most $O\left(C D(\log X)^{-\eta}\right)$ exceptions of $E_{c, d}$.

The particular case of $q=1$ of Corollary 3 improves [1, Theorem 6 ] by reducing the exponent $1 / 3$ to $13 / 40$. His exponent $1 / 3$ was obtained by a trivial treatment of the corresponding error term in Bantle \& Grupp's method. However for $q=1$, 
Kowalski, Robert \& Wu can get a better exponent 10/33 ([18], Theorem 1 with $\rho=\frac{1}{2}$ ), by Wu's method [28] with other ideas. The key of this method is to use triple exponential sums of type I instead of type II as in Bantle \& Grupp's method and that we can estimate sums of type I more effectively.

1.2. Signs of Hecke eigenvalues in short intervals. Let $k \geqslant 2$ be an even integer and $N \geqslant 1$ a squarefree integer. Let $f \in \mathcal{S}_{k}^{*}(N)$. In view of Sato-Tate's conjecture and the multiplicativity of $\lambda_{f}(n)$, it would be expected that

$$
\mathscr{N}_{f}^{ \pm}(x):=\sum_{\substack{n \leqslant x \\ \lambda_{f}(n) \gtrless 0}} 1 \gg_{f} x
$$

for all $x \geqslant x_{0}(f)$. Recently Kohnen, Lau \& Shparlinski ([17], Theorem 1) proved

$$
\mathscr{N}_{f}^{ \pm}(x) \gg_{f} \frac{x}{(\log x)^{17}}
$$

for $x \geqslant x_{0}(f)$. Then the exponent 17 was reduced to $1-1 / \sqrt{3}$ by $\mathrm{Wu}[29]$. Finally Lau \& Wu [20] established (1.11).

By coupling (1.12) with (1.9), Alkan and Zaharescu [17] showed that there are absolute constants $\vartheta<1$ and $A>0$ such that for any $f \in \mathcal{S}_{k}^{*}(N)$ the inequality

$$
\mathscr{N}_{f}^{ \pm}\left(x+x^{\vartheta}\right)-\mathscr{N}_{f}^{ \pm}(x)>0
$$

holds for $x \geqslant k^{A}$, but no explicit value of $\vartheta$ is evaluated. It is an interesting and important problem to know how small $\vartheta$ can be. The estimate (1.13) implies immediately that $\lambda_{f}(n)$ has sign-changes in short interval $\left[x, x+x^{\vartheta}\right]$ for all sufficiently large $x$. Lau \& $\mathrm{Wu}[20]$ succeeded in obtaining the first quantitative result: for any newform $f \in \mathcal{S}_{k}^{*}(N)$, there exists an absolute constant $C>0$ such that for all sufficiently large $x \geqslant N^{2} x_{0}(k)$, we have

$$
\mathscr{N}_{f}^{ \pm}\left(x+C N^{2+\varepsilon} x^{1 / 2}\right)-\mathscr{N}_{f}^{ \pm}(x) \gg_{\varepsilon}(N x)^{1 / 4-\varepsilon} .
$$

We are concerned with the sign changes of $\lambda_{f}(n)$ in the interval $\left[x, x+x^{\vartheta}\right]$ for $\vartheta<1 / 2$. In this case it seems rather difficult to establish the inequality (1.15) below for all large $x$. However, we can prove the following partial results. For simplicity, we consider only the case of $N=1$. In this case, we note $\mathcal{S}_{k}^{*}=\mathcal{S}_{k}^{*}(1)$. The next results can be easily generalized to the case of $\mathcal{S}_{k}^{*}(N)$.

Theorem 2. Let $\sigma=+$ or - . Suppose that $f \in \mathcal{S}_{k}^{*}$ and $3 / 7<\vartheta<1 / 2$. Then there exist two positive constants $C_{i}=C_{i}(f, \vartheta)(i=1,2)$ such that for any large parameter $T$, there are at least $C_{1} T^{1-\vartheta}(\log T)^{2}$ disjoint subintervals of length $C_{2} T^{\vartheta}(\log T)^{-2}$ in $[T, 2 T]$, such that the inequality

$$
\mathscr{N}_{f}^{\sigma}\left(x+x^{\vartheta}\right)-\mathscr{N}_{f}^{\sigma}(x) \gg_{f, \vartheta, \varepsilon} x^{\vartheta / 2-\varepsilon}
$$

holds, whenever $x$ lies in any of these subintervals. Moreover, we have

$$
\left|\left\{x \in[T, 2 T]: \mathscr{N}_{f}^{\sigma}\left(x+x^{\vartheta}\right)-\mathscr{N}_{f}^{\sigma}(x) \gg_{f, \vartheta, \varepsilon} x^{\vartheta / 2-\varepsilon}\right\}\right| \gg_{f, \vartheta, \varepsilon} T
$$

for all sufficiently large $T$.

Our approach of Theorem 2 is a variant of the method of Heath-Brown \& Tsang [13]. The main new ingredient is the estimate on the fourth moments of

$$
S_{f}(x):=\sum_{n \leqslant x} \lambda_{f}(n) .
$$


in short intervals (cf. Corollary 4 of Section 5), which can proved by using the method of Tanigawa \& Zhai [25]. From our proof of Theorem 2 given in Section 6 , it is easy to see that Conjecture 2 stated in Section 5 implies (1.15) for any $0<\vartheta<1 / 2$ (i.e. Conjecture 1 below). This conjecture is very strong, since it is an analogue of Jutila's conjecture (5.20) and Jutila has pointed out his conjecture implies the sixth power moment of the Riemann zeta-function $\zeta(s)$ over the critical line $\Re$ e $s=1 / 2$.

Theorem 3. Let $\sigma=+$ or - . Suppose that $f \in \mathcal{S}_{k}^{*}$ and $3 / 8 \leqslant \vartheta \leqslant 3 / 7$. Then there exist two positive constants $C_{i}=C_{i}(f, \vartheta)(i=3,4)$ such that for any large parameter $T$, there are at least $C_{3} T^{1-\vartheta}$ disjoint subintervals of length $C_{4} T^{\vartheta-\varepsilon}$ in $[T, 2 T]$, such that the inequality

$$
\mathscr{N}_{f}^{\sigma}\left(x+x^{\vartheta}\right)-\mathscr{N}_{f}^{\sigma}(x) \gg_{f, \vartheta, \varepsilon} x^{\vartheta / 2-2 \varepsilon}
$$

holds, whenever $x$ lies in any of these subintervals. Moreover, we have

$$
\left|\left\{x \in[T, 2 T]: \mathscr{N}_{f}^{\sigma}\left(x+x^{\vartheta}\right)-\mathscr{N}_{f}^{\sigma}(x) \gg_{f, \vartheta, \varepsilon} x^{\vartheta / 2-2 \varepsilon}\right\}\right| \gg_{f, \vartheta, \varepsilon} T^{1-\varepsilon}
$$

for all sufficiently large $T$.

In view of (1.14) and Theorems 2-3, it seems reasonable to propose the following conjecture.

Conjecture 1. Let $f \in \mathcal{S}_{k}^{*}$ and $0<\vartheta<1 / 2$. Then for any $\varepsilon>0$, the inequality (1.15) holds for $x \geqslant x_{0}(f, \vartheta, \varepsilon)$.

The next theorem shows that this conjecture is true for infinitely many $x$.

Theorem 4. Suppose that $f \in \mathcal{S}_{k}^{*}$ and $0<\vartheta<1 / 2$. Then for any $\varepsilon>0$, the inequality (1.15) holds for infinitely many $x$.

\section{A SPECIAL TRIPLE EXPONENTIAL SUM}

The next result is one of keys in the proofs of Theorem 1 and Propositions 2-3 below (see Sections 3-4).

Proposition 1. Let $\chi(h):=\mathrm{e}(\xi h)(\xi \in \mathbb{R}),\left|\varphi_{m}\right| \leqslant 1,\left|\psi_{n}\right| \leqslant 1$ and

$$
S:=\sum_{h \sim H} \sum_{m \sim M} \sum_{n \sim N} \chi(h) \varphi_{m} \psi_{n} \mathrm{e}\left(\frac{x h}{m n}\right) .
$$

Then for any $1 \leqslant H \leqslant M N y^{-1} x^{3 \varepsilon}$, we have

$$
S \ll_{\varepsilon} M N x^{-2 \varepsilon},
$$

provided one of the following conditions is satisfied:

$$
\begin{array}{cll}
x^{17 / 38} \leqslant y \leqslant x^{23 / 50}, & M \leqslant y x^{-\varepsilon^{\prime}}, & N \leqslant y^{10 / 14} x^{-3 / 14-\varepsilon^{\prime}} ; \\
x^{83 / 188} \leqslant y \leqslant x^{17 / 38}, & M \leqslant y x^{-\varepsilon^{\prime}}, & N \leqslant y^{14 / 12} x^{-5 / 12-\varepsilon^{\prime}} ; \\
x^{10 / 27} \leqslant y \leqslant x^{83 / 188}, & M \leqslant y x^{-\varepsilon^{\prime}}, & N \leqslant y^{27 / 19} x^{-10 / 19-\varepsilon^{\prime}} ; \\
x^{\kappa /(2 \kappa-\lambda+1)} \leqslant y \leqslant x^{(\kappa+1) /(2 \kappa-\lambda+2)}, & M \leqslant y x^{-\varepsilon^{\prime}}, & N \leqslant y^{(\kappa-\lambda+1) / 2} x^{-\kappa / 2-\varepsilon^{\prime}} ;
\end{array}
$$

where $(\kappa, \lambda)$ is an exponent pair. 
Proof. By [30, Theorem 2], we can write

$$
\begin{aligned}
S \ll & M N\left\{\left(x^{5} y^{-16} M^{2} N^{12}\right)^{1 / 26}+\left(x y^{-4} M N^{3}\right)^{1 / 6}\right. \\
& +\left(x^{20} y^{-56} M^{2} N^{38}\right)^{1 / 90}+\left(x^{3} y^{-12} M^{2} N^{14}\right)^{1 / 20} \\
& \left.+\left(x^{7} y^{-22} M N^{22}\right)^{1 / 36}+\left(x^{7} y^{-28} M^{6} N^{26}\right)^{1 / 44}+y^{-1} M+y^{-1} N^{2}\right\} x^{\varepsilon} .
\end{aligned}
$$

Thus the inequality (2.1) holds provided

$$
\begin{aligned}
& M \leqslant y x^{-\varepsilon^{\prime}}, \quad N^{12} \leqslant y^{14} x^{-5-\varepsilon^{\prime}}, \quad N^{3} \leqslant y^{3} x^{-1-\varepsilon^{\prime}}, \quad N^{19} \leqslant y^{27} x^{-10-\varepsilon^{\prime}}, \\
& N^{14} \leqslant y^{10} x^{-3-\varepsilon^{\prime}}, \quad N^{22} \leqslant y^{21} x^{-7-\varepsilon^{\prime}}, \quad N^{26} \leqslant y^{22} x^{-7-\varepsilon^{\prime}}, \quad N^{2} \leqslant y x^{-\varepsilon^{\prime}} .
\end{aligned}
$$

Among the above eight inequalities, it is easy to verify that for $x^{17 / 38} \leqslant y \leqslant x^{23 / 50}$, the fifth inequality implies all others except the first one. This proves (2.2). Assertions (2.3) and (2.4) can be treated similarly.

In order to prove (2.5), we apply Lemma 3.1 of [21] with the choice of parameters $(H, M, N)=(M, H, N), f(h)=h^{-1}, \alpha=1$ and $\beta=-1$ to write

$$
\begin{gathered}
S \ll M N\left\{\left(x^{\kappa} y^{-2 \kappa-2} M^{\lambda+1} N^{2}\right)^{1 /(2 \kappa+2)}+y^{-1} M\right. \\
\left.+\left(y^{-2} M N^{2}\right)^{1 / 2}+(x y)^{-1 / 2} M N\right\} x^{\varepsilon} .
\end{gathered}
$$

Thus the inequality (2.1) holds provided

$$
M \leqslant y x^{-\varepsilon^{\prime}}, \quad N^{2} \leqslant y^{2 \kappa-\lambda+1} x^{-\kappa-\varepsilon^{\prime}}, \quad N^{2} \leqslant y x^{-\varepsilon^{\prime}}, \quad N^{2} \leqslant y^{-1} x^{1-\varepsilon^{\prime}} .
$$

For $x^{\kappa /(2 \kappa-\lambda+1)} \leqslant y \leqslant x^{(\kappa+1) /(2 \kappa-\lambda+2)}$, it is easy to see that the second inequality implies the third and fourth. The proof of Proposition 1 is complete.

\section{3. $\mathscr{B}$-FREE NUMBERS IN SHORT PROGRESSIONS}

The distribution of $\mathscr{B}$-free numbers in short progressions was first studied by Alkan \& Zaharescu [2]. Their result is as follows: For all $\varepsilon>0, x \geqslant x_{0}(\mathscr{B}, \varepsilon)$, $y \geqslant x^{9 / 20+(1803 / 10) \varepsilon}$ and $1 \leqslant a \leqslant q \leqslant x^{\varepsilon}$ with $(a, q)=1$, we have

$$
\mid\{x<n \leqslant x+y: n \equiv a(\bmod q) \text { and } n \text { is } \mathscr{B} \text {-free }\} \mid \gg_{\mathscr{B}, \varepsilon} y / q .
$$

The particular case of (3.1) with $q=1$ was obtained by Bantle \& Grupp [5]. In [2,3], Alkan \& Zaharescu used the method of Bantle \& Grupp with some straightforward modification. The only difference is that they cannot directly apply Fouvry-Iwaniec's estimate on exponential sums ([11], Theorem 6) - the key tool in Bantle \& Grupp's method, because of the coefficient $\mathrm{e}\left(h a \bar{d}_{\sigma} \bar{p}_{1} \bar{p}_{2} / q\right)$ (see (C) below), which depends on the variables $p_{i}$ (and is equal to 1 if $q=1$ ). Since $q \leqslant x^{\varepsilon}$, it is easy to see that after a simple congruence argument (as Alkan \& Zaharescu remarked in [2], [3]), Fouvry-Iwaniec's estimate on exponential sums is still applicable.

Here we shall insert our new estimate on exponential sums into the method of Bantle \& Grupp with Alkan \& Zaharescu's adaptation to obtain a better result.

Proposition 2. For all $\varepsilon>0, x \geqslant x_{0}(\varepsilon), y \geqslant x^{17 / 38+100 \varepsilon}$ and $1 \leqslant a \leqslant q \leqslant x^{\varepsilon}$ with $((a, q), b)=1$ for all $b \in \mathscr{B}$, the inequality (3.1) holds.

Since Alkan \& Zaharescu's proof contains some inaccuracies (for example, (17) of [2] and [3] is inexact), we reproduce here the proof of Bantle \& Grupp with some simple adaptation, for the convenience of reader. 
The notation of this section is independent. Let $\theta, \delta_{1}$ and $\delta_{2}$ be some parameters such that

$$
\left\{\begin{array}{l}
\frac{1}{4}+\varepsilon \leqslant \theta<\frac{1}{2} \\
5 \varepsilon<\delta_{2}+4 \varepsilon<\delta_{1}+3 \varepsilon<\theta \\
\delta_{1}+\delta_{2}<1 \\
\delta_{1}+\delta_{2}+\theta-\varepsilon>1
\end{array}\right.
$$

Introducing

$$
\left\{\begin{array}{l}
\mathscr{P}_{1}:=\left\{p \in \mathscr{P}: x^{\delta_{1}}<p \leqslant x^{\delta_{1}+\varepsilon}\right\}, \\
\mathscr{P}_{2}:=\left\{p \in \mathscr{P}: x^{\delta_{2}}<p \leqslant x^{\delta_{2}+\varepsilon}\right\} .
\end{array}\right.
$$

Bantle \& Grupp's weight function is defined by

$$
c(n):=\sum_{\substack{p_{1} \in \mathscr{P}_{1} \\ p_{1} p_{2} \mid n}} \sum_{p_{2} \in \mathscr{P}_{2}} 1 .
$$

For $x \geqslant 3$ and $x \geqslant y \geqslant x^{\theta}$, put

$$
A:=\sum_{\substack{x<n \leqslant x+y \\ n \equiv a(\bmod q) \\ b \nmid n(\forall b \in \mathscr{B})}} c(n) .
$$

From (3.2), (3.3) and (3.4), it is easy to see that

$$
c(n) \leqslant\left(\delta_{1} \delta_{2}\right)^{-1} \quad(n \leqslant 2 x),
$$

which implies

$$
\sum_{\substack{x<n \leqslant x+y \\ n \equiv a(\bmod q) \\ b \nmid n(\forall b \in \mathscr{B})}} 1 \geqslant \delta_{1} \delta_{2} A .
$$

Thus it is sufficient to show that

$$
A \gg_{\mathscr{B}, \varepsilon} y / q .
$$

For this, we let $\ell:=\ell(\mathscr{B}, \varepsilon) \in \mathbb{N}$ be a positive integer such that

$$
\sum_{k=\ell+1}^{\infty} \frac{1}{b_{k}}<\frac{B \varepsilon^{3}}{\delta_{1} \delta_{2}}
$$

where

$$
B:=\prod_{b \in \mathscr{B}}\left(1-\frac{1}{b}\right)
$$

is the natural density of the sequence of $\mathscr{B}$-free numbers.

Clearly we can write

$$
A \geqslant A_{1}-A_{2}-A_{3},
$$


where

$$
\begin{aligned}
& A_{1}:=\sum_{\substack{x<n \leqslant x+y \\
n \equiv a(\bmod q) \\
b_{i} \nmid n(\forall i \leqslant \ell)}} c(n), \\
& A_{2}:=\sum_{\substack{b_{\ell}<b \leqslant y / q \\
b \in \mathscr{B}}} \sum_{\substack{x<n \leqslant x+y \\
n \equiv a(\bmod q) \\
b \mid n}} c(n), \\
& A_{3}:=\sum_{\substack{q<b \leqslant x \\
b \in \mathscr{B}}} \sum_{\substack{x<n \leqslant x+y \\
n \equiv a(\bmod q) \\
b \mid n}} c(n) .
\end{aligned}
$$

Lemma 3.1. We have

$$
A_{2} \leqslant \frac{2 B \varepsilon^{3}}{\delta_{1} \delta_{2}} \frac{y}{q}
$$

Proof. By (3.6), it follows that

$$
A_{2} \leqslant \frac{1}{\delta_{1} \delta_{2}} \sum_{\substack{b_{\ell}<b \leqslant y / q \\ b \in \mathscr{B}}} \sum_{\substack{x<n \leqslant x+y \\ n \equiv a(\bmod q) \\ n \equiv 0(\bmod b)}} 1
$$

Since $((a, q), b)=1$ for all $b \in \mathscr{B}$, by the Chinese Remainder Theorem we easily see that the system of equations $n \equiv a(\bmod q), n \equiv 0(\bmod b)$ has solution if and only if $(b, q)=1$. In this case, there is some integer $a^{\prime} \in\{1, \ldots, b q-1\}$ such that $n \equiv a^{\prime}(\bmod b q)$. Thus

$$
A_{2} \leqslant \frac{1}{\delta_{1} \delta_{2}} \sum_{\substack{b_{\ell}<b \leqslant y / q \\ b \in \mathscr{B}}}\left(\frac{y}{b q}+1\right) \leqslant \frac{1}{\delta_{1} \delta_{2}} \sum_{\substack{b_{\ell}<b \leqslant y / q \\ b \in \mathscr{B}}} \frac{2 y}{b q}
$$

which implies the required inequality in view of (3.8).

Lemma 3.2. There is a constant $C(\mathscr{B}, \varepsilon)$ such that

$$
A_{3} \leqslant \frac{C(\mathscr{B}, \varepsilon)}{\log x} \frac{y}{q}
$$

Proof. According to the definition of $c(n)$, we can write

$$
A_{3}=\sum_{\substack{y / q<b \leqslant x \\ b \in \mathscr{B}}} \sum_{p_{1} \in \mathscr{P}_{1}} \sum_{p_{2} \in \mathscr{P}_{2}} \sum_{\substack{x<n \leqslant x+y \\ n \equiv a(\bmod q) \\ n \equiv 0(\bmod b) \\ n \equiv 0\left(\bmod p_{1} p_{2}\right)}} 1 .
$$

As before, the system of equations $n \equiv a(\bmod q), n \equiv 0(\bmod b)$ has solution if and only if $(b, q)=1$. In this case, there is $a^{\prime} \in\{1, \ldots, b q-1\}$ such that $n \equiv a^{\prime}(\bmod b q)$. Since $b q>y$, the sum over $n$ is at most 1 . In this case, we must have $p_{1} \mid b$ or $p_{2} \mid b$, otherwise we have $n \equiv 0\left(\bmod b p_{1} p_{2}\right)$. Since $b p_{1} p_{2} \geqslant x^{\theta-\varepsilon+\delta_{1}+\delta_{2}}>2 x$ (in view of 
$(3.2))$ and $n \leqslant 2 x$, this is contradictory. Thus we deduce

$$
\begin{aligned}
A_{3} \leqslant & \sum_{\substack{y / q<b \leqslant x \\
b \in \mathscr{B}}} \sum_{p_{1} \in \mathscr{P}_{1}} \sum_{p_{2} \in \mathscr{P}_{2} \mid b} \sum_{\substack{x<n \leqslant x+y \\
n \equiv a(\bmod q) \\
n \equiv 0(\bmod b) \\
n \equiv 0\left(\bmod p_{1} p_{2}\right)}} 1 \\
& +\sum_{\substack{y / q<b \leqslant x \\
b \in \mathscr{B}}} \sum_{p_{1} \in \mathscr{P}_{1}} \sum_{p_{2} \in \mathscr{P}_{2}} \sum_{\substack{x<n \leqslant x+y \\
p_{2} \mid b=a(\bmod q) \\
n \equiv 0(\bmod b) \\
n \equiv 0\left(\bmod p_{1} p_{2}\right)}} 1 .
\end{aligned}
$$

Clearly

$$
\sum_{p_{i} \in \mathscr{P}_{i}} \sum_{\substack{x<n \leqslant x+y \\ n \equiv a(\bmod q) \\ n=0(\bmod b) \\ n \equiv 0\left(\bmod p_{1} p_{2}\right)}} 1 \leqslant \delta_{i}^{-1} \quad(i=1,2) .
$$

Inserting it into the preceding inequality, we find that

$$
\begin{aligned}
A_{3} & \leqslant \delta_{2}^{-1} \sum_{p_{1} \in \mathscr{P}_{1}} \sum_{\substack{y / q<b \leqslant x \\
b \in \mathscr{B}, p_{1} \mid b}} 1+\delta_{1}^{-1} \sum_{p_{2} \in \mathscr{P}_{2}} \sum_{\substack{y / q<b \leqslant x \\
b \in \mathscr{B}, p_{2} \mid b}} 1 \\
& \leqslant\left(\delta_{1}^{-1}+\delta_{2}^{-1}\right)\left|\mathscr{P}_{1}\right|,
\end{aligned}
$$

where we have used the hypothesis $\left(b_{i}, b_{j}\right)=1$ with $i \neq j$. This implies the required inequality.

For each $\sigma=\left\{k_{1}, \ldots, k_{i}\right\} \subset\{1, \ldots, \ell\}$, write $|\sigma|=i$ and $d_{\sigma}=b_{k_{1}} b_{k_{2}} \cdots b_{k_{i}}$ with the convention $|\emptyset|=0$ and $d_{\emptyset}=1$, where $\emptyset$ denotes the empty set.

Lemma 3.3. For $x \geqslant x_{0}(\mathscr{B}, \varepsilon)$, we have

$$
A_{1} \geqslant \frac{B \varepsilon^{2}}{2}(y / q)
$$

provided

$$
\left\{\begin{array}{l}
\frac{25}{58}<\theta<\frac{23}{50} \\
\delta_{1} \leqslant \theta-100 \varepsilon \\
\delta_{2} \leqslant \min \{(14 \theta-5) / 12,(10 \theta-3) / 14\}-100 \varepsilon .
\end{array}\right.
$$

Proof. Since $\left(b_{i}, b_{j}\right)=1(i \neq j)$, we can write

$$
\begin{aligned}
A_{1} & =\sum_{\sigma \subset\{1, \ldots, \ell\}}(-1)^{|\sigma|} \sum_{\substack{x<n \leqslant x+y \\
n \equiv a(\bmod q) \\
d_{\sigma} \mid n}} c(n) \\
& =\sum_{\substack{\sigma \subset\{1, \ldots, \ell\} \\
\left(d_{\sigma}, q\right)=1}}(-1)^{|\sigma|} \sum_{p_{1} \in \mathscr{P}_{1}} \sum_{p_{2} \in \mathscr{P}_{2}} \sum_{\substack{x<n \leqslant x+y \\
n \equiv a(\bmod q) \\
n \equiv 0\left(\bmod d_{\sigma} p_{1} p_{2}\right)}} 1 .
\end{aligned}
$$

In the second equality, we have used the following facts:

(i) $\left(d_{\sigma}, p_{1} p_{2}\right)=1$ for all $\sigma \subset\{1, \ldots, \ell\}$ and $p_{i} \in \mathscr{P}_{i}(i=1,2)$,

(ii) $n \equiv a(\bmod q)$ and $n \equiv 0\left(\bmod d_{\sigma} p_{1} p_{2}\right)$ have solution $\Leftrightarrow\left(d_{\sigma}, q\right)=1$. 
Since $\left(d_{\sigma} p_{1} p_{2}, q\right)=1$, there are $\bar{d}_{\sigma}, \bar{p}_{1}, \bar{p}_{2} \in\{1, \ldots, q-1\}$ such that

$$
d_{\sigma} \bar{d}_{\sigma} \equiv 1(\bmod q) \quad \text { and } \quad p_{i} \bar{p}_{i} \equiv 1(\bmod q) \quad(i=1,2) .
$$

It follows that

$$
A_{1}=\sum_{\substack{\sigma \subset\{1, \ldots, \ell\} \\\left(d_{\sigma}, q\right)=1}}(-1)^{|\sigma|} A_{1}(\sigma)
$$

where

$$
A_{1}(\sigma):=\sum_{p_{1} \in \mathscr{P}_{1}} \sum_{p_{2} \in \mathscr{P}_{2}} \sum_{\substack{x<d_{\sigma} p_{1} p_{2} m \leqslant x+y \\ m \equiv a^{*}(\bmod q)}} 1
$$

and $a^{*}:=a \bar{d}_{\sigma} \bar{p}_{1} \bar{p}_{2} \in\left\{1, \ldots,(q-1)^{4}\right\}$.

Introduce a function $\Phi(t) \in C^{\infty}(\mathbb{R})$ such that

$$
\begin{aligned}
\Phi(t)=1 & \text { if } t \in(x, x+y], \\
\Phi(t)=0 & \text { if } t \notin\left(x-y x^{-2 \varepsilon}, x+y+y x^{-2 \varepsilon}\right], \\
0 \leqslant \Phi(t) \leqslant 1 & \text { otherwise, }
\end{aligned}
$$

and

$$
\Phi^{(j)}(t) \ll\left(y x^{-2 \varepsilon}\right)^{-j} \quad(j \geqslant 0, t \in \mathbb{R}) .
$$

Thus we can write

$$
\begin{aligned}
A_{1}(\sigma) & =\sum_{p_{1} \in \mathscr{P}_{1}} \sum_{p_{2} \in \mathscr{P}_{2}} \sum_{m \equiv a^{*}(\bmod q)} \Phi\left(d_{\sigma} p_{1} p_{2} m\right)+O\left(y x^{-2 \varepsilon}\right) \\
& =\sum_{p_{1} \in \mathscr{P}_{1}} \sum_{p_{2} \in \mathscr{P}_{2}} \sum_{h \in \mathbb{Z}} \phi(h)+O\left(y x^{-2 \varepsilon}\right)
\end{aligned}
$$

where $\phi(t):=\Phi\left(a^{*} d_{\sigma} p_{1} p_{2}+t d_{\sigma} p_{1} p_{2} q\right)$. Let $A_{1}^{*}(\sigma)$ be the last sum. By Poisson's formula [26, Théorème I.6.1], we obtain

$$
A_{1}^{*}(\sigma)=\sum_{p_{1} \in \mathscr{P}_{1}} \sum_{p_{2} \in \mathscr{P}_{2}} \sum_{h \in \mathbb{Z}} \widehat{\phi}(h),
$$

where

$$
\widehat{\phi}(h)=\int_{\mathbb{R}} \phi(t) \mathrm{e}^{-2 \pi \mathrm{i} h t} \mathrm{~d} t=\frac{\mathrm{e}^{2 \pi \mathrm{i} h a^{*} / q}}{d_{\sigma} p_{1} p_{2} q} \widehat{\Phi}\left(\frac{h}{d_{\sigma} p_{1} p_{2} q}\right) .
$$

Thus

$$
A_{1}^{*}(\sigma)=\frac{1}{d_{\sigma} q} \sum_{p_{1} \in \mathscr{P}_{1}} \sum_{p_{2} \in \mathscr{P}_{2}} \frac{1}{p_{1} p_{2}} \sum_{h \in \mathbb{Z}} \mathrm{e}^{2 \pi \mathrm{i} h a^{*} / q} \widehat{\Phi}\left(\frac{h}{d_{\sigma} p_{1} p_{2} q}\right) .
$$

We shall evaluate $A_{1}^{*}(\sigma)$ by estimating the contribution to it according to

$$
h=0, \quad|h|>H:=y^{-1} x^{\delta_{1}+\delta_{2}+10 \varepsilon}, \quad|h| \leqslant H,
$$

respectively. 
(A) The contribution with $h=0$

Since

$$
\widehat{\Phi}(0)=\int_{\mathbb{R}} \Phi(t) \mathrm{d} t=y+O\left(y x^{-2 \varepsilon}\right),
$$

the contribution with $h=0$ to $A_{1}^{*}(\sigma)$ is equal to

$$
\left\{1+O\left(\frac{1}{x^{\varepsilon}}\right)\right\} \frac{y}{d_{\sigma} q} \sum_{p_{1} \in \mathscr{P}_{1}} \frac{1}{p_{1}} \sum_{p_{2} \in \mathscr{P}_{2}} \frac{1}{p_{2}} .
$$

(B) The contribution with $|h|>H:=y^{-1} x^{\delta_{1}+\delta_{2}+10 \varepsilon}$

By partial integration and (3.14), it follows that

$$
\begin{aligned}
\widehat{\Phi}\left(\frac{h}{d_{\sigma} p_{1} p_{2} q}\right) & =\left(\frac{2 \pi \mathrm{i} h}{d_{\sigma} p_{1} p_{2} q}\right)^{-j} \int_{\mathbb{R}} \Phi^{(j)}(t) \mathrm{e}^{-2 \pi \mathrm{i} t h /\left(d_{\sigma} p_{1} p_{2} q\right)} \mathrm{d} t \\
& \ll_{j}\left(\frac{d_{\sigma} p_{1} p_{2} q x^{2 \varepsilon}}{h y}\right)^{j} y .
\end{aligned}
$$

Thus the contribution with $|h|>H$ is, with the choice of $j=1 /(5 \varepsilon)+3$,

$$
\ll_{j} \frac{y}{q}\left(\frac{x^{\delta_{1}+\delta_{2}+5 \varepsilon}}{H y}\right)^{j} H \ll_{j} \frac{y}{q} x^{-5 j \varepsilon} H \ll_{\varepsilon} \frac{y}{q} x^{-5 \varepsilon} .
$$

(C) The contribution with $1 \leqslant|h| \leqslant H$

This contribution is

$$
\begin{aligned}
& =\frac{1}{d_{\sigma} q} \int_{\mathbb{R}} \Phi(t) \sum_{1 \leqslant|h| \leqslant H} \sum_{p_{1} \in \mathscr{P}_{1}} \sum_{p_{2} \in \mathscr{P}_{2}} \frac{\mathrm{e}^{2 \pi \mathrm{i} h a^{*} / q}}{p_{1} p_{2}} \mathrm{e}^{-2 \pi \mathrm{i} t h /\left(d_{\sigma} p_{1} p_{2} q\right)} \mathrm{d} t \\
& =\frac{1}{d_{\sigma} q} \int_{\mathbb{R}} \Phi(t) \sum_{1 \leqslant a_{1} \leqslant q} \sum_{1 \leqslant a_{2} \leqslant q} \sum_{1 \leqslant|h| \leqslant H} \sum_{\substack{p_{1} \in \mathscr{P}_{1} \\
\bar{p}_{1} \equiv a_{1}(\bmod q) \bar{p}_{2} \equiv a_{2}(\bmod q)}} \sum_{\substack{p_{2} \in \mathscr{P}_{2} \\
p_{1} p_{2}}} \frac{\chi(h)}{p_{1}} \mathrm{e}\left(\frac{-t h}{d_{\sigma} p_{1} p_{2} q}\right) \mathrm{d} t,
\end{aligned}
$$

where $\chi(h)=\mathrm{e}\left(h \bar{d}_{\sigma} a_{1} a_{2} / q\right)$ is an additive character independent of $p_{1}$ and $p_{2}$.

Define

$$
S_{t / d_{\sigma} q}\left(H^{\prime}, M, N\right):=\sum_{h \sim H^{\prime}} \sum_{m \sim M} \sum_{n \sim N} \chi(h) \varphi_{m} \psi_{n} \mathrm{e}\left(\frac{-\left(t /\left(d_{\sigma} q\right)\right) h}{p_{1} p_{2}}\right),
$$

where

$$
\begin{aligned}
\varphi_{m} & := \begin{cases}M m^{-1} & \text { if } m=p_{1} \in \mathscr{P}_{1} \text { and } \bar{p}_{1} \equiv a_{1}(\bmod q), \\
0 & \text { otherwise }\end{cases} \\
\psi_{n}: & = \begin{cases}N n^{-1} & \text { if } n=p_{2} \in \mathscr{P}_{2} \text { and } \bar{p}_{2} \equiv a_{2}(\bmod q), \\
0 & \text { otherwise }\end{cases}
\end{aligned}
$$


After dyadic split and using Proposition 1 to estimate $S_{t /\left(d_{\sigma} q\right)}\left(H^{\prime}, M, N\right)$, we can see that the contribution with $1 \leqslant|h| \leqslant H$ to $A_{1}^{*}(\sigma)$ is

$$
\begin{aligned}
& \ll \frac{1}{q} \int_{\mathbb{R}} \Phi(t) q^{2} \max _{\substack{1 \leqslant a_{1}, a_{2} \leqslant q \\
x / 2 \leqslant t \leqslant 2 x, 1 \leqslant H^{\prime} \leqslant H}} \frac{\left|S_{t /\left(d_{\sigma} q\right)}\left(H^{\prime}, M, N\right)\right|}{M N} \mathrm{~d} t \\
& \ll \frac{1}{q} \int_{\mathbb{R}} \Phi(t) q^{2} x^{-4 \varepsilon} \mathrm{d} t \\
& \ll \frac{y}{q} x^{-\varepsilon},
\end{aligned}
$$

provided (3.11) is satisfied.

Combining all, we obtain that

$$
A_{1}(\sigma)=\left\{1+O\left(\frac{1}{x^{\varepsilon}}\right)\right\} \frac{y}{d_{\sigma} q} \sum_{p_{1} \in \mathscr{P}_{1}} \frac{1}{p_{1}} \sum_{p_{2} \in \mathscr{P}_{2}} \frac{1}{p_{2}}+O_{\mathscr{B}, \varepsilon}\left(\frac{y}{q} x^{-\varepsilon}\right) .
$$

Now the required result follows from (3.12) by noticing that

$$
\begin{aligned}
\sum_{\substack{\sigma \subset\{1, \ldots, \ell\} \\
\left(d_{\sigma}, q\right)=1}} \frac{(-1)^{|\sigma|}}{d_{\sigma}} & =\prod_{\substack{i=1 \\
\left(b_{i}, q\right)=1}}^{\ell}\left(1-\frac{1}{b_{i}}\right) \geqslant B \\
\sum_{p_{i} \in \mathscr{P}_{i}} \frac{1}{p_{i}} & =\log \left(\frac{\delta_{i}+\varepsilon}{\delta_{i}}\right)+O\left(\frac{1}{\log x}\right) \geqslant \varepsilon
\end{aligned}
$$

for $x \geqslant x_{0}(\mathscr{B}, \varepsilon)$.

Now we are ready to complete the proof of Proposition 2. Take

$$
\theta=\frac{17}{38}, \quad \delta_{1}=\theta-\varepsilon^{\prime}, \quad \delta_{2}=1-2 \theta+\varepsilon^{\prime} .
$$

It is easy to verify that these choices satisfy the conditions (3.2) and (3.11). Thus Lemmas 3.1, 3.2 and 3.3 imply that

$$
A \geqslant\left(\frac{B \varepsilon^{2}}{2}-\frac{B \varepsilon^{3}}{\delta_{1} \delta_{2}}-\frac{2 C(\mathscr{B}, \varepsilon)}{\log x}\right) \frac{y}{q} \gg_{\mathscr{B}, \varepsilon} \frac{y}{q}
$$

for $x \geqslant x_{0}(\mathscr{B}, \varepsilon)$. This completes the proof of (3.7) and hence Proposition 2.

\section{Special $\mathscr{B}$-Free numbers AND Proof of Theorem 1}

Let $\mathfrak{P} \subset \mathscr{P}$ be a set of prime numbers satisfying the following condition

$$
|\mathfrak{P} \cap[1, x]| \ll_{f} x^{\rho} \frac{(\log \log x)^{\Psi_{\rho}}}{(\log x)^{\Theta_{\rho}}} \quad(x \geqslant 2),
$$

where $\rho \in[0,1]$ and $\Theta_{\rho}, \Psi_{\rho}$ are real constants such that $\Theta_{1}>1$. In this section, we consider a special set

$$
\mathscr{B}_{\mathfrak{P}}:=\mathfrak{P} \cup\left\{p^{2}: p \in \mathscr{P} \backslash \mathfrak{P}\right\} .
$$

Clearly $\mathscr{B}_{\mathfrak{P}}$ verifies the condition (1.6) 
Proposition 3. Let $\mathscr{B}_{\mathfrak{P}}$ be defined as above and let

$$
\theta(\rho)= \begin{cases}29 \rho /(46 \rho+19) & \text { if } 190 / 323<\rho \leqslant 166 / 173 \\ 17 \rho /(26 \rho+12) & \text { if } 166 / 173<\rho \leqslant 1\end{cases}
$$

or

$$
\theta(\rho)=\frac{(\kappa+2) \rho}{(\kappa-\lambda+3) \rho+2} \text { for } \quad \frac{\kappa}{\kappa-\lambda+1} \leqslant \rho \leqslant 1,
$$

where $(\kappa, \lambda)$ is an exponent pair. Then for any $\varepsilon>0, x \geqslant x_{0}(\mathfrak{P}, \varepsilon), y \geqslant x^{\theta(\rho)+\varepsilon}$ with and $1 \leqslant a \leqslant q \leqslant x^{\varepsilon}$ with $(a, q)=1$, we have

$$
\mid\left\{x<n \leqslant x+y: n \equiv a(\bmod q) \text { and } n \text { is } \mathscr{B}_{\mathfrak{P}}-\text { free }\right\} \mid \gg_{\mathfrak{P}, \varepsilon} y / q \text {. }
$$

The proof of this proposition is very similar to that of Proposition 2. We shall only point out some different points.

Let $\rho, \theta, \delta_{1}$ and $\delta_{2}$ be some parameters such that

$$
\left\{\begin{array}{l}
\frac{1}{4}+\varepsilon \leqslant \theta<\frac{1}{2} \\
4 \varepsilon<\delta_{2}+3 \varepsilon<\delta_{1}+2 \varepsilon<(\theta-\varepsilon) / \rho \\
\delta_{1}+\delta_{2}<1 \\
\delta_{1}+\delta_{2}+\theta / \rho>1
\end{array}\right.
$$

Similarly define $A^{\prime}, A_{1}^{\prime}, A_{2}^{\prime}, A_{3}^{\prime}$ by replacing $\mathscr{B}$ by $\mathscr{B}_{\mathfrak{P}}$. Thus we have

$$
A^{\prime} \geqslant A_{1}^{\prime}-A_{2}^{\prime}-A_{3}^{\prime} \text {. }
$$

As a particular case of Lemma 3.1, we have

$$
A_{2}^{\prime} \leqslant \frac{B_{\mathfrak{P}} \varepsilon^{3}}{\delta_{1} \delta_{2}} \frac{y}{q}
$$

where

$$
B_{\mathfrak{P}}:=\prod_{p \in \mathfrak{P}}\left(1-\frac{1}{p}\right) \prod_{p \in \mathscr{P} \backslash \mathfrak{P}}\left(1-\frac{1}{p^{2}}\right) .
$$

We shall use the method of [18] to estimate $A_{3}^{\prime}$. An advantage of this method is to allow us to relax $\delta_{1}+\varepsilon<\theta$ (for general $\left.\mathscr{B}\right)$ to $\delta_{1}+\varepsilon<(\theta-\varepsilon) / \rho$ (for $\mathscr{B}_{\mathfrak{P}}$ ).

Lemma 4.1. There is a constant $C(\mathfrak{P}, \varepsilon)$ such that

$$
A_{3}^{\prime} \leqslant \frac{C(\mathfrak{P}, \varepsilon)}{(\log x)^{1 / 2}} \frac{y}{q} \quad(x \geqslant 2) .
$$

Proof. According to the definition of $\mathscr{B}_{\mathfrak{P}}$, we can write

$$
A_{3}^{\prime}=A_{3,1}^{\prime}+A_{3,2}^{\prime}+A_{3,3}^{\prime}+A_{3,4}^{\prime} \text {, }
$$


where

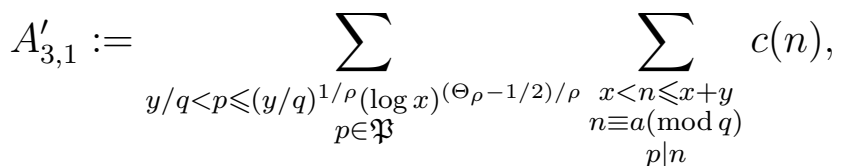

$$
\begin{aligned}
& A_{3,2}^{\prime}:=\sum_{(y / q)^{1 / \rho}(\log x)^{\left(\Theta_{\rho}-1 / 2\right) / \rho}<p \leqslant x} \sum_{\substack{x<n \leqslant x+y \\
p \in a(\bmod q) \\
p \mid n}} c(n), \\
& A_{3,3}^{\prime}:=\sum_{\substack{y<p^{2} \leqslant(y / q)^{2} \\
p \notin \mathfrak{P}}} \sum_{\substack{x<n \leqslant x+y \\
n \equiv a(\bmod q) \\
p^{2} \mid n}} c(n),
\end{aligned}
$$

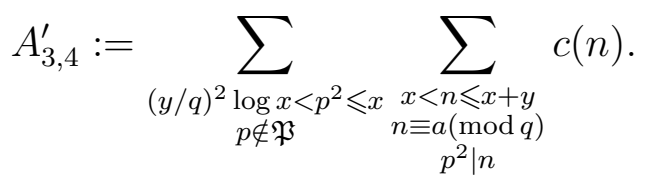

In $A_{3,1}^{\prime}$, we have $(p, q)=1$. Thus there is some integer $a^{\prime} \in\{1, \ldots, p q-1\}$ such that $n \equiv a^{\prime}(\bmod p q)$. Since $p q>y$, the sum over $n$ is at most 1 . Thus For $p>y$, there is at most an integer $n \in(x, x+y]$ such that $p \mid n$. Thus

$$
\begin{aligned}
A_{3,1}^{\prime} & \leqslant\left(\delta_{1} \delta_{2}\right)^{-1} \sum_{\substack{p \leqslant(y / q)^{1 / \rho}(\log x)^{\left(\Theta_{\rho}-1 / 2\right) / \rho} \\
p \in \mathfrak{P}}} 1 \\
& \ll \frac{\left((y / q)^{1 / \rho}(\log x)^{\left(\Theta_{\rho}-1 / 2\right) / \rho}\right)^{\rho}}{(\log x)^{\Theta_{\rho}}} \\
& \ll \frac{(y / q)}{(\log x)^{1 / 2}} .
\end{aligned}
$$

The definition of $c(n)$ allows us to write

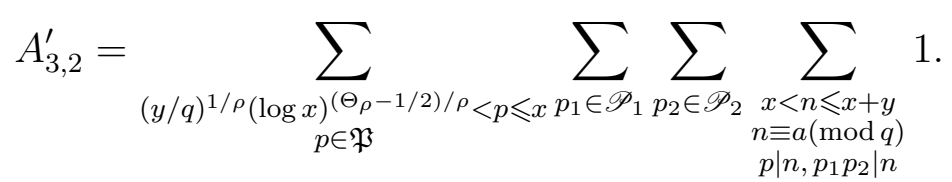

The hypothesis $\delta_{2}+2 \varepsilon<\delta_{1}+\varepsilon<(\theta-\varepsilon) / \rho$ and $p \in \mathfrak{P}$ imply $\left(p, p_{1} p_{2}\right)=1$. Thus $p p_{1} p_{2} \mid n$. Since $p p_{1} p_{2}>x^{\theta / \rho+\delta_{1}+\delta_{2}}(\log x)^{\left(\Theta_{\rho}-1 / 2\right) / \rho} \geqslant 2 x$, the sum over $n$ must be empty. Therefore $A_{3,2}^{\prime}=0$.

We have

$$
A_{3,3}^{\prime} \leqslant \frac{1}{\delta_{1} \delta_{2}} \sum_{\substack{q \leqslant(y / q)(\log x)^{1 / 2} \\ p \in \mathscr{P} \backslash \mathfrak{P}}} 1 \ll \frac{(y / q)}{(\log x)^{1 / 2}} .
$$

The term $A_{3,4}^{\prime}$ will be treated by the method of Filaseta \& Trifonov [10]. Defining

$$
S\left(t_{1}, t_{2}\right):=\left\{d \in\left(t_{1}, t_{2}\right]: \text { there is an integer } k \text { such that } k d^{2} \in(x, x+y]\right\},
$$


we can deduce, in view of (3.6), that

$$
\begin{aligned}
A_{3,4}^{\prime} & \leqslant\left(\delta_{1} \delta_{2}\right)^{-1} \sum_{\substack{(y / q)^{2} \log x<p^{2} \leqslant x \\
p \in \mathscr{P} \backslash \mathfrak{P}}} \sum_{\substack{x<n \leqslant x+y \\
p^{2} \mid n}} 1 \\
& \leqslant\left(\delta_{1} \delta_{2}\right)^{-1}\left|S\left(y q^{-1}(\log x)^{1 / 2}, x^{1 / 2}\right)\right| .
\end{aligned}
$$

We split $\left(y q^{-1}(\log x)^{1 / 2}, x^{1 / 2}\right]$ into dyadic intervals $\left(x^{\phi}, 2 x^{\phi}\right]$ and write

$$
A_{3,4}^{\prime} \leqslant\left(\delta_{1} \delta_{2}\right)^{-1}(\log x) \max _{\theta-\varepsilon \leqslant \phi \leqslant 1 / 2}\left|S\left(x^{\phi}, 2 x^{\phi}\right)\right| .
$$

According to $[10,(4)]$, we have

$$
\left|S\left(x^{\phi}, 2 x^{\phi}\right)\right| \ll x^{(1-\phi) / 3}
$$

for $y q^{-1}(\log x)^{1 / 2} \leqslant x^{\phi} \leqslant 2 x^{1 / 2}$, and thus infer with the hypothesis $\theta>\frac{1}{4}+3 \varepsilon$ that

$$
A_{3,4}^{\prime} \ll(y / q) x^{-\varepsilon} .
$$

Now inserting these estimates into (4.6), we obtain the required inequality.

Now we are ready to complete the proof of Proposition 3.

Take

$$
\theta=\theta(\rho), \quad \delta_{1}=\theta-\varepsilon^{\prime}, \quad \delta_{2}=1-(1+1 / \rho) \theta+\varepsilon^{\prime} .
$$

It is easy to verify that these choices satisfy the conditions (4.3). Thus Lemma 3.3, (4.5) and Lemma 4.1 imply that

$$
A \geqslant\left(B_{\mathfrak{P}} \varepsilon^{2}-\frac{B_{\mathfrak{P}} \varepsilon^{3}}{\delta_{1} \delta_{2}}-\frac{2 C(\mathfrak{P}, \varepsilon)}{(\log x)^{1 / 2}}\right) \frac{y}{q} \gg_{\mathfrak{P}, \varepsilon} \frac{y}{q}
$$

for $x \geqslant x_{0}(\mathfrak{P}, \varepsilon)$. This completes the proof of (3.7) and hence Proposition 3.

\section{Moments of $S_{f}(x)$ in short intervals}

Recall the definition (1.16):

$$
S_{f}(x):=\sum_{n \leqslant x} \lambda_{f}(n)
$$

This section will be devoted to investigate the $2 \ell$ th-moment $(\ell=1,2)$

$$
\mathcal{M}_{\ell}(T, U):=\int_{T}^{2 T}\left|S_{f}(x+U)-S_{f}(x)\right|^{2 \ell} \mathrm{d} x,
$$

which is a principal tool for the proof of Theorems 2-3, where $T \geqslant 1$ and $U \geqslant 1$ are large parameters.

When $\ell=1$, this type of integral was studied by Good [12] for $E(t)$, which is defined by

$$
E(t):=\int_{0}^{t}|\zeta(1 / 2+\mathrm{i} u)|^{2} \mathrm{~d} u-t \log (t / 2 \pi)-(2 \gamma-1) t \quad(t \geqslant 2),
$$

and by Jutila [16] for $\Delta(x)$ which is the error term of the Dirichlet divisor problem, given by the formula

$$
\Delta(x)=\sum_{n \leqslant x} \tau(n)-x(\log x+2 \gamma-1)
$$


Here $\zeta(s)$ denotes the function of Riemann, $\tau(n)$ is the classical divisor function and $\gamma$ the Euler constant.

Jutila [16] first studied the mean square of the difference $\Delta(x+U)-\Delta(x)$ for short intervals. He proved that if $T \geqslant 2$ and $1 \leqslant U \ll T^{1 / 2} \ll H \leqslant T$, then

$$
\begin{aligned}
& \int_{T}^{T+H}|\Delta(x+U)-\Delta(x)|^{2} \mathrm{~d} x \\
& =\frac{1}{4 \pi^{2}} \sum_{n \leqslant T /(2 U)} \frac{\tau(n)^{2}}{n^{3 / 2}} \int_{T}^{T+H} x^{1 / 2}\left|\mathrm{e}\left((n / x)^{1 / 2} U\right)-1\right|^{2} \mathrm{~d} x \\
& \quad+O\left(T^{1+\varepsilon}+H U^{1 / 2} T^{\varepsilon}\right),
\end{aligned}
$$

with the notation $\mathrm{e}(t):=\mathrm{e}^{2 \pi \mathrm{i} t}(t \in \mathbb{R})$, which implies that the estimate

$$
\int_{T}^{T+H}|\Delta(x+U)-\Delta(x)|^{2} \mathrm{~d} x \asymp H U\left(\log \left(T^{1 / 2} / U\right)\right)^{3}
$$

holds for $T \geqslant 2, H U \gg T^{1+\varepsilon}$ and $T^{\varepsilon} \ll U \leqslant T^{1 / 2} / 2$. For $E(t)$, Jutila also obtained similar results.

When $H=T$, Ivić [14] substantially improved Jutila's asymptotic formulas. For $\Delta(x)$, Ivić proved for $T^{\varepsilon} \ll U \leqslant T^{1 / 2} / 2$ that

$$
\begin{aligned}
\int_{T}^{2 T}|\Delta(x+U)-\Delta(x)|^{2} \mathrm{~d} x= & T U \sum_{j=0}^{3} c_{j}(\Delta)\left(\log \left(T^{1 / 2} / U\right)\right)^{j} \\
& +O_{\varepsilon}\left(T^{1 / 2+\varepsilon} U^{2}+T^{1+\varepsilon} U^{1 / 2}\right)
\end{aligned}
$$

where the $c_{j}(\Delta)$ are constants.

Jutila [16] conjectured that the estimates

$$
\begin{gathered}
\int_{T}^{2 T}|\Delta(x+U)-\Delta(x)|^{4} \mathrm{~d} x \ll_{\varepsilon} T^{1+\varepsilon} U^{2}, \\
\int_{T}^{2 T}|E(t+U)-E(t)|^{4} \mathrm{~d} x \ll_{\varepsilon} T^{1+\varepsilon} U^{2}
\end{gathered}
$$

are true for $1 \ll U \ll T^{1 / 2}$. These two conjectures are very strong since Jutila pointed out that (5.6) implies the important conjectural bound

$$
\int_{1}^{T}|\zeta(1 / 2+\mathrm{i} t)|^{6} \mathrm{~d} t \ll_{\varepsilon} T^{1+\varepsilon}
$$

Ivić [14] proved that (5.5) holds for $T^{3 / 8} \ll U \ll T^{1 / 2}$. The term $T^{1+\varepsilon} U^{2}$ in (5.5) is best possible with respect to $T^{\varepsilon}$. However, when $T^{3 / 7} \ll U \ll T^{1 / 2}$, Tanigawa and Zhai [25] proved an asymptotic formula for the integral

$$
\int_{T}^{2 T}|\Delta(x+U)-\Delta(x)|^{4} \mathrm{~d} x .
$$

The proofs of the above estimates of $\Delta(x)$ are all based on the well-known truncated Voronoi's formula

$$
\Delta(x)=\frac{x^{1 / 4}}{\pi \sqrt{2}} \sum_{n \leqslant N} \frac{\tau(n)}{n^{3 / 4}} \cos \left(4 \pi \sqrt{n x}-\frac{\pi}{4}\right)+O\left(x^{\varepsilon}+N^{-1 / 2} x^{1 / 2+\varepsilon}\right),
$$

where $1<N \ll x^{A}, A>0$ is an arbitrarily large constant. 
For $S_{f}(x)$ we have the following similar Voronoi's formula

$$
S_{f}(x)=\frac{x^{1 / 4}}{\pi \sqrt{2}} \sum_{n \leqslant M} \frac{\lambda_{f}(n)}{n^{3 / 4}} \cos \left(4 \pi \sqrt{n x}-\frac{\pi}{4}\right)+O_{f, \varepsilon}\left(x^{\varepsilon}+M^{-1 / 2} x^{1 / 2+\varepsilon}\right)
$$

for $1 \ll M \ll x^{A}$. Besides the analogy between (5.7) and (5.8), we also have the the bound (1.3). So the proofs of the above estimates apply also to $S_{f}(x)$ without modifications.

When $\ell=1$, Ivić $[14]$ proved the following

Proposition 4. For each $f \in \mathcal{S}_{k}^{*}$, there is a positive constant $C(f)>0$ such that for any $\varepsilon>0$ the asymptotic formula

$$
\mathcal{M}_{1}(T, U)=C(f) T U+O_{f, \varepsilon}\left(T^{3 / 5+\varepsilon} U^{9 / 5}+T^{1+\varepsilon} U^{1 / 2}\right)
$$

holds uniformly for $T \geqslant 1$ and $T^{\varepsilon} \ll U \leqslant T^{1 / 2} / 2$. In particular we have

$$
\frac{1}{2} C(f) T U \leqslant \mathcal{M}_{1}(T, U) \leqslant 2 C(f) T U,
$$

uniformly for $T \geqslant T_{0}(f, \varepsilon)$ and $T^{\varepsilon} \leqslant U \leqslant T^{1 / 2-\varepsilon}$, where $T_{0}(f, \varepsilon)$ is a suitable positive constant depending on $f$ and $\varepsilon$ only.

When $\ell=2$, Ivić's argument for $\int_{T}^{2 T}|\Delta(x+U)-\Delta(x)|^{4} \mathrm{~d} x$ yields the following

Proposition 5. For all $f \in \mathcal{S}_{k}^{*}$ and any $\varepsilon>0$, we have

$$
\mathcal{M}_{2}(T, U) \ll_{f, \varepsilon} T^{1+\varepsilon} U^{2}+T^{7 / 4+\varepsilon} .
$$

uniformly for $T \geqslant 1$ and $T^{\varepsilon} \ll U \ll T^{1 / 2}$. Especially when $T^{3 / 8} \ll U \ll T^{1 / 2}$, we have

$$
\mathcal{M}_{2}(T, U) \ll_{f, \varepsilon} T^{1+\varepsilon} U^{2} .
$$

If applying the argument of Tanigawa and Zhai [25] to $S_{f}(x)$, we have

Proposition 6. For each $f \in \mathcal{S}_{k}^{*}$ there is a small positive constant $c=c_{f}$ such that for any $\varepsilon>0$ we have

$$
\begin{aligned}
\mathcal{M}_{2}(T, U)= & \frac{3}{\pi^{4}} \int_{T}^{2 T} x\left(\sum_{n \leqslant c(T / U)^{1 / 4}} \frac{\lambda_{f}(n)^{2}}{n^{3 / 2}} \sin ^{2}(\pi U \sqrt{n / x})\right)^{2} \mathrm{~d} x \\
& +O_{f, \varepsilon}\left(T^{1 / 2+\varepsilon} U^{3}+T^{19 / 16+\varepsilon} U^{25 / 16}+T^{17 / 8+\varepsilon} U^{-5 / 8}\right) .
\end{aligned}
$$

uniformly for $T \geqslant 1$ and $T^{3 / 7} \ll U \ll T^{1 / 2}$.

From Proposition 6 we can prove the following

Corollary 4. For all $f \in \mathcal{S}_{k}^{*}$ and any $\varepsilon>0$, we have

$$
\mathcal{M}_{2}(T, U) \ll_{f, \varepsilon} T U^{2} .
$$

uniformly for $T \geqslant 1$ and $T^{3 / 7+\varepsilon} \ll U \ll T^{1 / 2-\varepsilon}$.

Proof. With the help of trivial inequality $|\sin t| \leqslant \min \{|t|, 1\}$ and Rankin-Selberg's well known result (cf. [22])

$$
\sum_{n \leqslant x}\left|\lambda_{f}(n)\right|^{2}=A(f) x+O\left(x^{3 / 5}\right)
$$


where $A(f)$ is the positive constant depending on $f$, a simple partial summation shows that the integral in (5.13) is

$$
\begin{aligned}
& \ll T \int_{T}^{2 T}\left(\sum_{n \leqslant T / U^{2}} \frac{\lambda_{f}(n)^{2}}{n^{3 / 2}} \frac{U^{2} n}{x}\right)^{2} \mathrm{~d} x+T \int_{T}^{2 T}\left(\sum_{T / U^{2}<n \leqslant c(T / U)^{1 / 4}} \frac{\lambda_{f}(n)^{2}}{n^{3 / 2}}\right)^{2} \mathrm{~d} x \\
& \ll T U^{2} .
\end{aligned}
$$

This proves the required result.

Analogous to Jutila's conjectures (5.5) and (5.6), we may propose the following

Conjecture 2. The estimate (5.14) holds uniformly for $T^{\varepsilon} \ll U \ll T^{1 / 2}$.

For $\Delta(x)$, Tong [27] established the well-known asymptotic formula

$$
\int_{1}^{T}|\Delta(x)|^{2} \mathrm{~d} x=C T^{3 / 2}+F(T)
$$

with $F(T) \ll T(\log T)^{5}$. Recently, Lau \& Tsang [19] proved

$$
F(T) \ll T(\log T)^{3} \log \log T .
$$

Ivić [15] proved that

$$
\int_{1}^{T}\left|S_{f}(x)\right|^{2} \mathrm{~d} x=C(f) T^{3 / 2}+O_{f}\left(T(\log T)^{5}\right) .
$$

Combining the approaches of [15] and [19], it is easy to show

$$
\mathcal{M}_{1}(T, U) \ll_{f} T U+T \log T
$$

for $1 \leqslant U \ll T^{1 / 2}$.

Heath-Brown \& Tsang [13] proved that if $2 \leqslant H \leqslant T^{1 / 2} / 2$, then

$$
\int_{T}^{2 T} \max _{0 \leqslant h \leqslant H}|\Delta(x+h)-\Delta(x)|^{2} \mathrm{~d} x \ll T H(\log T)^{5} .
$$

Applying their approach to the function $S_{f}(x)$ with the help of (5.18) we get the following result.

Proposition 7. For each $f \in \mathcal{S}_{k}^{*}$, we have

$$
\int_{T}^{2 T} \max _{0 \leqslant h \leqslant H}\left|S_{f}(x+h)-S_{f}(x)\right|^{2} \mathrm{~d} x \ll_{f} T H(\log T)^{2},
$$

uniformly for $2 \leqslant H \leqslant T^{1 / 2} / 2$.

Proof. Write $H=2^{\lambda} b$ with $\lambda \in \mathbb{N}$ and $1 \leqslant b<2$. Similar to the proof of [13, Lemma 2 ], we can deduce by using (5.18) that the integral in (5.20) is

$$
\begin{aligned}
& \ll \lambda \sum_{\mu \leqslant \lambda} \sum_{0 \leqslant \nu<2^{\mu}} \int_{T+v 2^{\lambda-\mu} b}^{T+v 2^{\lambda-\mu} b}\left|S_{f}\left(x+2^{\lambda-\mu} b\right)-S_{f}(x)\right|^{2} \mathrm{~d} t+T(\log T)^{2} \\
& \ll \lambda \sum_{\mu \leqslant \lambda} \sum_{0 \leqslant \nu<2^{\mu}}\left(T 2^{\lambda-\mu} b+T \log T\right)+T(\log T)^{2} \\
& \ll \lambda^{2} H T+\lambda H T \log T+T(\log T)^{2} \\
& \ll H T(\log T)^{2} .
\end{aligned}
$$




\section{Proofs of Theorems 2 and 3}

In this section we shall prove Theorem 2 by using the method of Heath-Brown \& Tsang [13]. We only consider the case with sign "+". The proof for the case "-" is the same.

For $f \in \mathcal{S}_{k}^{*}, t \geqslant 1$ and $U \geqslant 1$, define

$$
\begin{aligned}
R_{U}(t) & :=S_{f}(t+U)-S_{f}(t)=\sum_{t<n \leqslant t+U} \lambda_{f}(n), \\
R_{U}^{+}(t ;) & :=\max \left\{R_{U}(t), 0\right\} .
\end{aligned}
$$

Lemma 6.1. Let $f \in \mathcal{S}_{k}^{*}$ and $\varepsilon>0$. Then we have

$$
\int_{T}^{2 T} R_{f}(t ; U) \mathrm{d} t \ll_{f, \varepsilon} T^{1+\varepsilon}
$$

uniformly for $U \ll T$.

Proof. Taking $M=T$ in (5.8), it follows that $(j=0,1)$

$$
\begin{aligned}
\int_{T}^{2 T} S_{f}\left(t+j T^{\vartheta}\right) \mathrm{d} t \ll & \sum_{n \leqslant M} \frac{\tau(n)}{n^{3 / 4}}\left|\int_{T}^{2 T}\left(t+j T^{\vartheta}\right)^{1 / 4} \cos \left(4 \pi \sqrt{n\left(t+j T^{\vartheta}\right)}-\frac{\pi}{4}\right) \mathrm{d} t\right| \\
& +T^{1+\varepsilon} .
\end{aligned}
$$

This and the first derivative test imply (6.3) immediately.

We only consider $T^{3 / 7+\varepsilon} \leqslant U \leqslant T^{1 / 2-\varepsilon}$, since the other case $T^{3 / 8+\varepsilon} \leqslant U \leqslant T^{3 / 7+\varepsilon}$ is similar. By the Hölder inequality and (5.10), we have

$$
T U \ll \int_{T}^{2 T}\left|R_{U}(t)\right|^{2 / 3+4 / 3} \mathrm{~d} t \ll\left(\int_{T}^{2 T}\left|R_{U}(t)\right| \mathrm{d} t\right)^{2 / 3}\left(\int_{T}^{2 T}\left|R_{U}(t)\right|^{4} \mathrm{~d} t\right)^{1 / 3} .
$$

Together with (5.12), it leads to

$$
\int_{T}^{2 T}\left|R_{U}(t)\right| \mathrm{d} t \gg T U^{1 / 2}
$$

and hence

by (6.3) and the relation

$$
\int_{T}^{2 T} R_{U}^{+}(t) \mathrm{d} t \gg T U^{1 / 2}
$$

$$
2 R_{U}^{+}(t)=R_{U}(t)+\left|R_{U}(t)\right|
$$

Let $\delta>0$ be a small constant. Then

$$
T U^{1 / 2} \ll \int_{R_{U}^{+}(t)>\delta U^{1 / 2}}^{2 T} R_{U}^{+}(t) \mathrm{d} t+\delta T U^{1 / 2} .
$$

So, for sufficiently small $\delta>0$, we have

$$
\int_{T}^{2 T} R_{U}^{+}(t) \mathrm{d} t \gg T U^{1 / 2} .
$$

With the Cauchy-Schwarz inequality, we deduce that

$$
T^{2} U \ll\left|\left\{t \in[T, 2 T]: R_{U}^{+}(t)>\delta U^{1 / 2}\right\}\right| \int_{T}^{2 T}\left|R_{U}(t)\right|^{2} \mathrm{~d} t .
$$


With (5.10) and the trivial inequality

$$
R_{U}^{+}(t) \ll T^{\varepsilon}\left(\mathscr{N}_{f}^{+}(t+U)-\mathscr{N}_{f}^{+}(t)\right),
$$

the second part of Theorem 2 follows readily.

In view of (6.4) and the trivial inequality ||$a|-| b|| \leqslant|a-b|$, we have

$$
\begin{aligned}
\left|R_{U}^{+}(t+h)-R_{U}^{+}(t)\right| & \leqslant\left|R_{U}(t+h)-R_{U}(t)\right| \\
& \leqslant\left|S_{f}(t+U+h)-S_{f}(t+U)\right|+\left|S_{f}(t+h)-S_{f}(t)\right| .
\end{aligned}
$$

Applying Proposition 7, it follows that, for $H(\log T)^{2} \leqslant \delta U$,

$$
\int_{T}^{2 T} \max _{0 \leqslant h \leqslant H}\left|R_{U}^{+}(t+h)-R_{U}^{+}(t)\right|^{2} \mathrm{~d} t \ll \delta T U,
$$

where $\delta>0$ is a small constant. By (6.5) and the Cauchy-Schwarz inequality, we deduce that

$$
\begin{gathered}
\int_{T}^{2 T}\left(R_{U}^{+}(t)-\max _{0 \leqslant h \leqslant H}\left|R_{U}^{+}(t+h)-R_{U}^{+}(t)\right|\right) \mathrm{d} t \gg T U^{1 / 2}, \\
\int_{T}^{2 T}\left(R_{U}^{+}(t)-\max _{0 \leqslant h \leqslant H}\left|R_{U}^{+}(t+h)-R_{U}^{+}(t)\right|\right)^{2} \mathrm{~d} t \ll T U,
\end{gathered}
$$

provided $H(\log T)^{2} \leqslant \delta U$. Repeating the argument in (6.5)-(6.6), we have

$$
\left|\left\{t \in[T, 2 T]: R_{U}^{+}(t)-\max _{0 \leqslant h \leqslant H}\left|R_{U}^{+}(t+h)-R_{U}^{+}(t)\right|>\delta U^{1 / 2}\right\}\right| \gg T .
$$

Take $U=T^{\theta}$ and $H=T^{\theta}(\log T)^{-2}$. We divide the interval [T, 2T] into subintervals of length $H$. In view of $(6.7)$, there are at least $O\left(T^{1-\theta}(\log T)^{-2}\right)$ subintervals containing a point $x$ such that

$$
R_{U}^{+}(x)-\max _{0 \leqslant h \leqslant H}\left|R_{U}^{+}(x+h)-R_{U}^{+}(x)\right|>\delta U^{1 / 2} .
$$

Since

$$
\min _{x \leqslant t \leqslant x+H} R_{U}^{+}(t) \geqslant R_{U}^{+}(x)-\max _{0 \leqslant h \leqslant H}\left|R_{U}^{+}(x+h)-R_{U}^{+}(x)\right|>\delta U^{1 / 2} .
$$

this implies that for all $t \in[x, x+H], R_{U}^{+}(t)>\delta U^{1 / 2}$ and hence

$$
\mathscr{N}_{f}^{+}(t+U)-\mathscr{N}_{f}^{+}(t) \gg \delta U^{1 / 2} T^{-\varepsilon} \text {. }
$$

Clearly, $[x, x+H]$ may overlap at most two subintervals, so we can select alternate subintervals to avoid overlapping. The proof of Theorem 2 is complete.

The proof of Theorem 3 is similar to that of Theorem 2. The essential difference is to apply Proposition 5 in place of Proposition 6. So we omit the details.

Remark 1. Ivić's estimate (5.4) implies that for any $0<\vartheta<1 / 2$ we have

$$
\Delta\left(x+T^{\vartheta}\right)-\Delta(x)=\Omega\left(T^{\vartheta / 2}(\log T)^{3 / 2}\right), \quad x \in[T, 2 T] .
$$

However, when $3 / 7<\vartheta<1 / 2$, our argument of this paper implies that

$$
\left|\left\{x \in[T, 2 T]: \pm\left(\Delta\left(x+T^{\vartheta}\right)-\Delta(x)\right) \gg T^{\vartheta / 2}(\log T)^{3 / 2}\right\}\right| \gg T,
$$

which is an improvement of (6.8). 


\section{Proof of Theorem 4}

From Proposition 4, there exists infinitely many $x$ such that

$$
\left|S_{f}\left(x+x^{\vartheta}\right)-S_{f}(x)\right| \gg x^{\vartheta / 2}
$$

which implies that

$$
\mathscr{N}_{f}^{+}\left(x+x^{\vartheta}\right)-\mathscr{N}_{f}^{+}(x) \gg x^{\vartheta / 2-\varepsilon / 5} .
$$

This proves (1.15) with the sign "+".

Let $p_{0}$ be the least prime such that $p_{0} \nmid N$ and $\lambda_{f}\left(p_{0}\right)<0$. Since each integer $n$ counted in (7.1) can be written as $n=p_{0}^{\nu} m$ with $p_{0} \nmid m$ and $0 \leqslant \nu \leqslant 4 \log x,(7.1)$ becomes

$$
\sum_{0 \leqslant \nu \leqslant 4 \log x} \sum_{\begin{array}{c}
x<p_{0}^{\nu} m \leqslant x+x^{\vartheta} \\
p_{0} \nmid m, \lambda_{f}\left(p_{0}^{\nu}\right) \lambda_{f}(m)>0
\end{array}} 1 \gg x^{\vartheta / 2-\varepsilon / 5} .
$$

Thus there is at least a $\nu \in \mathbb{N} \cap[0,4 \log x]$ such that

$$
\sum_{\substack{x<p_{0}^{\nu} m \leqslant x+x^{\vartheta} \\ p_{0} \nmid m, \lambda_{f}\left(p_{0}^{\nu}\right) \lambda_{f}(m)>0}} 1 \gg x^{\vartheta / 2-\varepsilon / 4} .
$$

From this we deduce that at least one of the following two inequalities

$$
\begin{aligned}
& \sum_{\substack{x / p_{0}^{\nu}<m \leqslant x / p_{0}^{\nu}+x^{\vartheta} / p_{0}^{\nu} \\
p_{0} \nmid m, \lambda_{f}(m)>0}} 1 \gg x^{\vartheta / 2-\varepsilon / 4} \\
& \sum_{\substack{x / p_{0}^{\nu}<m \leqslant x / p_{0}^{\nu}+x^{\vartheta} / p_{0}^{\nu} \\
p_{0} \nmid m, \lambda_{f}(m)<0}} 1 \gg x^{\vartheta / 2-\varepsilon / 4}
\end{aligned}
$$

holds. Clearly these imply that (1.15) with the sign "_" holds for $x / p_{0}^{\nu}$ or $x / p_{0}^{\nu-1}$ in place of $x$, respectively.

Acknowledgement. The authors deeply thank the referee for his/her careful reading of the manuscript and many valuable comments. In particular he/she suggested Theorem 4 and a simplified proof of Theorem 2 .

Funding. The second author is supported by National Natural Science Foundation of China (Grant No. 11171344) and Natural Science Foundation of Beijing (Grant No. 1112010).

\section{REFERENCES}

[1] E. Alkan, On the sizes of gaps in the Fourier expansion of modular forms, Canad. J. Math. 57 (2005), no. 3, 449-470.

[2] E. Alkan \& A. Zaharescu, B-free numbers in short arithmetic progressions, J. Number Theory 113 (2005), no. 2, 226-243.

[3] E. Alkan \& A. Zaharescu, Nonvanishing of Fourier coefficients of newforms in progressions, Acta Arith. 116 (2005), no. 1, 81-98.

[4] A. Balog \& K. Ono, The Chebotarev density theorem in short intervals and some questions of Serre, J. Number theory 91 (2001), 356-371.

[5] G. Bantle \& F. Grupp, On a problem of Erdős and Szemerédi, J. Number Theory 22 (1986), 280-288.

[6] C. David \& F. Pappalardi, Average Frobenius distributions of elliptic curves, Internat. Math. Res. Notices 4 (1999), 165-183. 
[7] P. Deligne, La conjecture de Weil, I, II, Publ. Math. IHES 48 (1974), 273-308, 52 (1981), 313-428.

[8] N. Elkies, Distribution of supersingular primes, Astérisque No. 198-200 (1991), 127-132.

[9] P. Erdös, On the difference of consecutive terms of sequences, defined by divisibility properties, Acta Arith. 12 (1966), 175-182.

[10] M. Filaseta \& O. Trifonov, On gaps between squarefree numbers II, J. London Math. Soc. (2) 45 (1992), 215-221.

[11] E. Fouvry \& H. Iwaniec, Exponential sums with monomials, J. Number Theory 33 (1989), 311-333.

[12] A. Good, Ein $\Omega$-Resultat für das quadratische Mittel delRiemannschen Zetafunktion auf der kritische Linie, Invent. Math. 41 (1977), 233-251.

[13] D. R. Heath-Brown \& K. Tsang, Sign changes of $E(t), \Delta(x)$ and $P(x)$, J. Number Theory 49 (1994), 73-83.

[14] A. Ivić, On the divisor function and the Riemann zeta-function in short intervals, Ramanujan J., 55(2009), 207-224.

[15] A. Ivić, Large values of certain number-theoretic error terms, Acta Arith. 56 (1990), 135-159.

[16] M. Jutila, On the divisor problem for short intervals, Ann. Univ. Turkuensis Ser. AI 186 (1984), 23-30.

[17] W. Kohnen, Y.-K. Lau \& I. E. Shparlinski, On the number of sign changes of Hecke eigenvalues of newforms, J. Austral. Math. Soc. 85 (2008), no. 1, 87-94.

[18] E. Kowalski, O. Robert \& J. Wu, Small gaps in coefficients of L-functions and $\mathscr{B}$-free numbers in short intervals, Revista Matemática Iberoamericana 23 (2007), No. 1, 281-322.

[19] Y.-K. Lau \& K.-M. Tsang, On the mean square formula of the error term in the Dirichlet divisor problem, Math. Proc. Camb. Phil. Soc. 146 (2009), no. 2, 277-287.

[20] Y.-K. Lau \& J. Wu, The number of Hecke Eigenvalues of same signs, Math. Z. 263 (2009), 957-970.

[21] H.-Q. Liu \& J. Wu, Numbers with a large prime factor, Acta Arith. 89 (1999), 163-187.

[22] R. A. Rankin, Contributions to the theory of Ramanujan's function $\tau(n)$ and similar arithmetical functions II. The order of the Fourier coefficients of integral modular forms, Proc. Cambridge Phil. Soc. 35 (1939), 357-372.

[23] J.-P. Serre, Quelques applications du théorème de densité de Chebotarev, Inst. Hautes Études Sci. Publ. Math. 54 (1981), 323-401.

[24] E. Szemerédi, On the difference of consecutive terms of sequences, defined by divisibility properties II, Acta Arith. 23 (1973), 359-361.

[25] Y. Tanigawa \& W. G. Zhai, On the fourth power moment of $\Delta(x)$ and $E(x)$ in short intervals, Int. J. Number Theory 5 (2009), no. 2, 355?382.

[26] G. Tenenbaum, Introduction à la théorie analytique et probabiliste des nombres, Cours Spécialisés $\mathrm{N}^{\circ}$ 1. Société Mathématique de France, Paris, 1995. xv+457 pp.

[27] K. C. Tong, On divisor problem III, Acta Math. Sinica 6 (1956), 515-541.

[28] J. Wu, Nombres $\mathscr{B}$-libres dans les petits intervalles, Acta Arith. 65 (1993), 97-116.

[29] J. Wu, Power sums of Hecke eigenvalues and application, Acta Arith. 137 (2009), 333-344.

[30] J. Wu, Almost primes in short intervals, Science in China Series A: Mathematics 53 (2010), No. 9, 2511-2524.

School of Mathematics, Shandong University, Jinan, Shandong 250100, China, Institut Elie Cartan Nancy, CNRS, Université Henri Poincaré (Nancy 1), INRIA, 54506 VANDGEUVRE-LÈS-NANCY, FRANCE

E-mail address: wujie@iecn.u-nancy.fr

Department of Mathematics, China University of Mining and Technology, BeiJING 100083, CHINA

E-mail address: zhaiwg@hotmail.com 